\title{
How to read small bowel capsule endoscopy: a practical guide for everyday use
}

\section{(ㄷ)(1) $\odot$}

Authors

Emanuele Rondonotti ${ }^{1}$, Marco Pennazio ${ }^{2}$, Ervin Toth ${ }^{3}$, Anastasios Koulaouzidis ${ }^{4}$

Institutions

1 Gastroenterology Unit, Ospedale Valduce, Como, Italy

2 University Division of Gastroenterology. City of Health and Science University Hospital, Turin, Italy

3 Department of Gastroenterology, Skane University Hospital, Lund University, Malmö, Sweden

4 Endoscopy Unit, The Royal Infirmary of Edinburgh, Edinburgh, Scotland

\author{
Bibliography \\ Endoscopy International Open 2020; 08: E1220-E1224 \\ DOI 10.1055/a-1210-4830 \\ ISSN 2364-3722
}

(c) 2020. The Author(s).

This is an open access article published by Thieme under the terms of the Creative Commons Attribution-NonDerivative-NonCommercial License, permitting copying and reproduction so long as the original work is given appropriate credit. Contents may not be used for commecial purposes, or adapted, remixed, transformed or built upon. (https://creativecommons.org/licenses/by-nc-nd/4.0/)

Corresponding author

Dr. Anastasios Koulaouzidis MD, DM, FEBG, FRCPE, FASGE, The Royal Infirmary of Edinburgh, Centre for Liver and Digestive Disorders, Endoscopy Unit, Little France Crescent

Fax: +441312421619

akoulaouzidis@hotmail.com
Small bowel (SB) capsule endoscopy (SBCE) is often perceived by both patients and physicians as a "lightweight," noninvasive, comfortable procedure that is easily performed with little more than swallowing a pill and a glass of water. However, "easy to carry out" does not translate to ease of reading or interpretation. A SB capsule acquires thousands of images and generates a long video; however, clinically relevant findings are often seen in only a few frames. There is no way to direct or focus the camera, the capsule's lens cannot be cleaned or luminal debris removed, and perhaps most importantly, the capsule cannot take tissue samples. Therefore, the rate of missed lesions in the SB has been quoted as high as $10 \%$ [1]. Despite various SB imaging modalities offering similar diagnostic yield (DY) and miss rates, SBCE is one of the few endoscopic procedures open to immense scrutiny, as the data recorded are readily accessible for further on-demand review. This may expose clinicians to additional litigation but conversely, also provides a unique learning platform for new generations of SBCE readers. This is certainly fertile ground for virtual artificial neural network (ANN) training systems and even artificial intelligence ( $\mathrm{Al}$ ) diagnosis [2]. Currently, several different SBCE platforms exist. They differ in technical features and specifications such as size and weight, number and position of cameras, frame rate acquisition, and battery duration, as well as in several functions of proprietary reading software. In this editorial, we aim to provide general principles for SBCE reading, which are only partially addressed in recent technical reviews or guidelines [3-9]. We would also like to suggest tips and tricks to reduce common deficiencies in SBCE.

\section{Reading capsule endoscopy:} when and where

The following paragraph describes the SBCE video review process. It is crucial to point out that, as emphasized in several clinical guidelines [3-5, 7-9] this is only one step of a complex diagnostic work-up. Before approaching any SBCE video, it is essential to be aware of clinical details about a patient, such as the duration of the clinical history, comorbidities, medications, and clinical presentation. Knowledge of these data allows for a thorough, focused, and fruitful SBCE revision process. It could be argued that knowing clinical data may introduce an anticipation bias, leading to overestimating the role of this procedure in the diagnostic work-up. However, knowledge of clinical data is crucial for a balanced evaluation of findings and meaningful SBCE conclusion.

Although there are obvious variations in anatomy, pathology, patient demographics, and technical specifications for each SBCE model, the capsule generally takes between 1.5 to 4 to 5 hours to cover the entire SB. This generates a video with a long run time and often prolonged sections of normal SB, which requires time, focused attention, and dedication to accurately evaluate and review $[6,10-12]$. It is a misconception that SBCE video reading is a simple task that can be undertaken as an activity performed in between other, more compelling clinical duties. In our opinion, SBCE reading should be afforded protected time and be performed as thoroughly and diligently as any other endoscopic activity. Experience can reduce SBCE reading time, however, the time allocated for review of one 


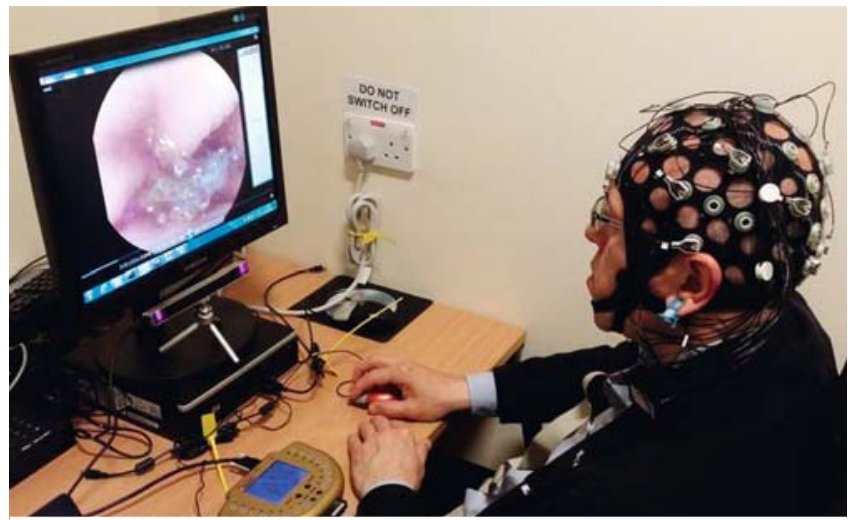

- Fig. 1 Author's unpublished work/data on eye tracking/electroencephalogram recording concentration during SBCE reading.

SBCE video should be at least 45 to 50 minutes even for an expert reader. This time slot includes short breaks, scheduled every 15 to 20 minutes, to maintain the reader's focused attention during the reviewing process [10-12].

It is fair to say that the SBCE reviewing process, especially in long and/or negative videos, can be monotonous and even tedious. However, the reader should remain vigilant to pick up even a single abnormal frame among thousands of normal frames. In our experience, the subconscious brain takes over to maximize performance, picking up subtle changes in texture or discoloration that under different circumstances, may not have been noted ( $\triangleright$ Fig. 1). A neutral background environment is suggested (i.e. quiet room, with adjustable/dimmable lights and possibly music) to assist concentration and minimize distraction [12]. Reading in a dim and quiet room may promote drowsiness, especially if the reading is performed at the end of a long working day. This reinforces the idea of identifying dedicated slots for SBCE reading, as a part of regular endoscopy activity. Seek advice from your occupational health team to create a proper environment, chair posture, and use of a computer monitor screen (over 21 "' is recommended), especially in highvolume services. A technique that may help to maintain focus is to keep a hand on the mouse, and a finger on the scroll wheel, as constant movements of the mouse and the fine-tuned control provided by the scroll wheel can help the reader remain constantly engaged with the video reading.

\section{Basics of review \& important steps in SB capsule endoscopy reading}

The first step in SBCE evaluation is a quick overview of the entire video. This can be done using specifically designed, automated fast-reading modes (e.g. QuickView, TOP 100, OmniMode, Duoview etc.) [13-15] or manual scrolling through the video at a high frame rate (near the maximum available frame rate), the latter of which is our personal preference. This first phase is to quickly identify possible areas of interest for further detailed review, to identify key anatomical landmarks (namely the pylorus and ileocecal valve) and more importantly to gain vital clinical information quickly in relevant circumstances, e.
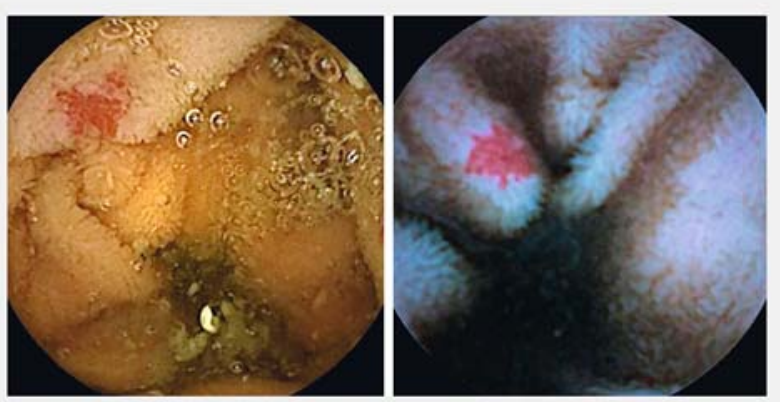

- Fig. 2 SBCE images of an angiectasia under white light (left; PillCamSB3) and Blue Mode reading (right; PillCamSB2).

g. investigation of gastrointestinal bleeding. Landmarking is fundamental for assessment of SB cleansing, confirmation of complete SB exploration, and lesion localization for application of standardized scoring. Following landmarking, we recommend proceeding directly to SB assessment. It is important to highlight a common issue with review speed: the capsule often resembles a "bullet train" in the proximal SB [16], where it has been clearly demonstrated that both vascular and neoplastic lesions are most frequently located [17], and slows down when passing though the distal SB. Therefore, regardless of the capsule model, we recommend decreasing the frame rate to 6 to 8 frames/second when inspecting the proximal SB. According to the reading protocol proposed by the European Society of Gastrointestinal Endoscopy [6] the frame rate can be increased when in the mid-distal SB. However, we suggest that readers avoid high frame rates (e.g. 16-20 frames/sec) in almost every SB segment, otherwise identification of minimal changes may be impossible even for an expert reader.

Use of dual-frame review for most frontal-view capsules has been widely adopted [6], but novice readers may wish to continue using the single frame mode or adopting a more personal style, should they wish, especially if it allows them to focus and enjoy the review. When reviewing the SB, we strongly advise saving any relevant findings to create a case library that can be reviewed quickly and easily; ( Fig.2). The second review should focus on comparing the saved thumbnails and relevant short video sections. Due to the propulsive activity of the SB, capsules often move bi-directionally, therefore, the same lesion or anomaly may appear several times; running through the library may also help estimate the number and location of findings. Each selected thumbnail should be compared with software-embedded Atlas images and with results of previous studies performed in the same center. The relevant ones should be annotated using dedicated classification or scoring systems (e.g. Niv score, Lewis score, CECDAl [Capsule Endoscopy Crohn's Disease Activity Index](, Saurin classification, SPICE [smooth, protruding lesion index on capsule endoscopy] score) [18-21]. The overall clinical evaluation of a SB video and report results from the combined evaluation of annotated thumbnails. Before finalizing the report, we suggest systematically checking the stomach and the colon, even if the capsule system in use is 
- Table 1 SAVER acronym for memory aid for capsule endoscopy report checklist.

S PICE any protrusion(s); Girelli index (mass of bulge)

A ssess lesion(s) potential for bleeding (Saurin Score)

$\checkmark \quad$ aluate inflammation (Lewis score or CECDAI)

E estimate the location of important lesion(s)/finding(s) (Li score)

$\mathrm{R} \quad$ ecord image \& overall video cleanliness

SPICE, smooth, protruding lesion index on capsule endoscopy; CECDAI, Capsule Endoscopy Crohn's Disease Activity Index.

not designed for other gastrointestinal segments, as obvious gastric and colonic lesions overlooked on initial gastroscopy and colonoscopy may be discovered in $7 \%$ to $15 \%$ (up to $30 \%$ in some reports) of patients [22]. This is particularly important in cases where evaluation of the SB is negative.

In addition, we suggest a "think twice" policy in negative SB exams; we recommend that the same reviewer, or where possible, a second, more experienced reviewer, repeat the complete review process. Last but not least, standardization is required and every report should contain quantifiable information. For this purpose, we suggest following the SAVER acronym; Specification of protrusions (i.e. SPICE score for masses/bulges) [18], Assessment of a lesions (especially angiectasias) bleeding potential (i.e. through the Saurin score) [19], eValuation of inflammatory activity (e.g. by Lewis or CECDAI cores) [20,21], Estimate of location of important lesions [23], and Recording of bowel/image cleanliness ( $>$ Table 1 ) [24].

\section{How to shorten video reading times}

Because SBCE reading is time-consuming, several options have been evaluated to save time without compromising lesion detection rate. The more obvious way to shorten reading time is to simply increase the reading frame rate. However, the impact of this approach on lesion detection is still unclear. While some studies have demonstrated low miss rates, we believe that increasing reading frame speed may lead to diagnostic error. Capsule manufacturers have introduced different software image analysis systems to automatically select or highlight relevant images and eliminate sequential images which are similar and redundant [13-15]. These systems can reduce reading time by up to $50 \%$ with a very low diagnostic miss rate; in our opinion, although they cannot substitute for a thorough video reading, they might represent a valid form of support. Similarly, automatic systems for selection of frames containing blood (or red areas) have been developed, which may show some promise in patients with suspected small bowel bleeding, but relevant frames can still be missed. Currently, we cannot rely heavily on these tools in the SBCE reading process. In addition, because capsule images are not of high resolution when compared to conventional endoscopes, any image enhancement tool (e.g. Fujinon Intelligent Chromo Endoscopy-FICE, and Blue Mode for PillCam, Augmented Live-body Image Color spectrum
- Table 2 Ten tips and tricks for an effective SBCE reading.

1. Get your training sorted! Achieving competency before starting SBCE reading is mandatory and maintaining proficiency thereafter is vital

2. Avoid CE reading if you are tired; it is a long and tough process requiring focused attention

3. Avoid CE reading in between other gastrointestinal procedures or tasks; instead, set up dedicated reading slots (45-60 min each)

4. Set up an inspiring and dedicated SBCE reading environment, i. e. quiet room with dimmable lights, big-sized screen etc.

5. Collect as much clinical data as possible before SBCE reading; you are not just describing findings; you are also participating in a diagnostic process

6. Follow a predefined sequence; pre-reading, landmarking, findings \& clips selection and reporting; don't forget to check gastrointestinal segments outside the SB

7. Slow down, whether you are an expert or a novice reader, and consider a slower reading speed in the proximal SB. Avoid high (e. g. 18-20 fps) speeds

8. Be proactive; move through the video and compare images or findings to other videos, online resources or in-print libraries

9. Set up a SB multidisciplinary team and whenever possible compare your findings with the results of other diagnostic procedures (e. g. device-assisted enteroscopy, surgery, radiology imaging). It is critical to reappraise both morphology and clinical relevance of SBCE findings

10. Enjoy reading CE or relinquish the task

SBCE, small bowel capsule endoscopy; CE: capsule endoscopy.

Enhancement-ALICE for Mirocam, Advanced Color Enhancement-ACE for CapsoCam etc.,) is welcome [25]. Currently available image enhancement tools seem to allow for slightly more accurate characterization of observed lesions, but they do not significantly improve the detection rate of clinically relevant lesions.

Ultimately, we believe that although SBCE is a highly technical tool, the most effective way to shorten reading times while maintaining accuracy and effectiveness is strictly linked to reader competency. Although expertise in upper and lower gastrointestinal endoscopy is necessary prerequisite $[9,26,27]$, SBCE has specific technical features that make it different from other endoscopy techniques (e.g. underwater view, non-distended lumen, tangential non-adjustable view, back-and-forth capsule movements etc.). Therefore, even experienced endoscopists still require dedicated training. However, SBCE training is currently not standardized, differing widely between countries and institutions [26, 27] and the definition of competency has not yet been universally established. Several clinical researchers have evaluated the number of SBCE procedures needed to obtain reading privileges. The Korean Gut Study Group and the American Society for Gastrointestinal Endoscopy define 10 and 25 SBCE readings, respectively, as thresholds for defining competency [9]; however, more recent well-designed 
studies using SBCE assessment tools conclude that basic competency can be obtained after 20 to 25 supervised videos. Advanced competency may require a more comprehensive training curriculum. Experience is a relative point in SBCE reading, and further exposure to a larger volume of reviewed SBCEs not only reduces reading time but also improves identification of subtle mucosal changes. However, even more important than a threshold number of SBCEs for achieving and maintaining competency is participation in a dedicated SB multidisciplinary working group. Only through continuous collaboration with specialists who perform other dedicated SB diagnostics or therapeutics can SBCE readers improve their ability to evaluate the lesions identified, for example, in estimating size and location. Ideally, SBCE readers should also be involved in device-assisted enteroscopy procedures (or at least see some of them). This helps to bridge the gap between wired and wireless endoscopy, ensuring a continuous reappraisal process that eventually improves SBCE reading. Participating in dedicated meetings in which findings or cases can be discussed with experts or in a peer-to-peer setting may also be helpful in maintaining competency.

- Table 2 lists 10 tips and tricks for an effective capsule endoscopy reading process.

\section{Conclusion}

In light of recent advances, and the unique nature of SBCE, we can expect an acceleration in technology. Al systems offer the ability to extract only relevant points of interest from the thousands of images generated and provide a preliminary report much like current electrocardiogram machines [28]. Until that time, training and expert guidance remain the cornerstone for competent clinical evaluation of SBCE videos.

\section{Acknowledgement}

The authors thank Dr. Alex Robertson for the thorough language review and essential editorial support.

\section{Competing interests}

Dr. Pennazio has received honoraria from Olympus and Medtronic. Dr. Toth has received honoraria and/or travel support from Olympus, Medtronic, Norgine and Ambu. Dr. Koulaouzidis has received material support for research from SynMed UK, IntroMedic and Aquilant; he also received the 2011 ESGE-Given Imaging Ltd research grant. He has received honoraria and/or travel support from Dr Falk Pharma, Jinshan, Ferring Pharmaceuticals UK and Norgine and has been on the advisory boards for Dr Falk Pharma UK, Tillotts Pharma, IntroMedic. Dr Koulaouzidis also is cofounder of AJM Med-i-Caps.

\section{References}

[1] Lewis BS, Eisen GM et al. A pooled analysis to evaluate results of capsule endoscopy trials. Endoscopy 2005; 37: 960-965

[2] Leenhardt R, Vasseur P, Li C et al. CAD-CAP Database Working Group. A neural network algorithm for detection of GI angioectasia during small-bowel capsule endoscopy. Gastrointest Endosc 2019; 89: 189194

[3] Pennazio M, Spada C, Eliakim R et al. Small-bowel capsule endoscopy and device-assisted enteroscopy for diagnosis and treatment of small-bowel disorders: European Society of Gastrointestinal Endoscopy (ESGE) Clinical Guideline. Endoscopy 2015; 47: 352-376

[4] Yamamoto H, Ogata H, Matsumoto T et al. Clinical Practice Guideline for Enteroscopy. Dig Endosc 2017; 29: 519-546

[5] Gerson LB, Fidler JL, Cave DR et al. ACG Clinical Guideline: Diagnosis and Management of Small Bowel Bleeding. Am J Gastroenterol 2015; 110: $1265-1287$

[6] Rondonotti E, Spada C, Adler S et al. Small-bowel capsule endoscopy and device-assisted enteroscopy for diagnosis and treatment of small-bowel disorders: European Society of Gastrointestinal Endoscopy (ESGE) Technical Review. Endoscopy 2018; 50: 423-446

[7] Enns RA, Hookey L, Armstrong D et al. Clinical practice guidelines for the use of video capsule endoscopy. Gastroenterology 2017; 152: 497-514

[8] Oliva S, Thomson M, de Ridder L et al. Endoscopy in Pediatric Inflammatory Bowel Disease: A Position Paper on Behalf of the Porto IBD Group of the European Society for Pediatric Gastroenterology, Hepatology and Nutrition. J Pediatr Gastroenterol Nutr 2018; 67: 414-430

[9] Faigel DO, Baron TH, Adler DG et al. ASGE. ASGE guideline: guidelines for credentialing and granting privileges for capsule endoscopy. Gastrointest Endosc 2005; 61: 503-505

[10] Lo S. How should we do capsule endoscopy reading? Tech Gastroint Endosc 2006; 8: 146-148

[11] Barkin JA, Barkin JS. Video capsule endoscopy teaching, reading and troubleshooting. Gastrointest Clin N Am 2017; 27: 15-27

[12] Lewis SB. How to read capsule endoscopy images. Gastrointest Clin N Am 2004; 14: 11-26

[13] Freitas M, Arieira C, Carvalho PB et al. Simplify to improve in capsule endoscopy - top 100 is a swift and reliable evaluation tool for the small bowel inflammatory activity in Crohn's disease. Scand J Gastroenterol 2020; 55: 408-413

[14] Saurin JC, Lapalus MG, Cholet F et al. Can we shorten the small-bowel capsule reading time with the "Quick-view" image detection system. Dig Liver Dis 2012; 44: 477-581

[15] Gomes C, Pinho R, Ponte A et al. Evaluation of the sensitivity of the Express View ${ }^{\circledR}$ in the Mirocam capsule endoscopy software. Scand J Gastroenterol 2020; 55: 371-375

[16] Worsoe J, Fynne L, Gregersen T et al. Gastric transit and small bowel transit time and motility assessed by a magnet tracking system. BMC Gastroenterol 2011; 29: 145

[17] Davie M, Yung DE, Douglas S et al. Mapping the distribution of small bowel angioectasias. Scand J Gastroenterol 2019; 54: 597-602

[18] Girelli CM, Porta P, Colombo E et al. Development of a novel index to discriminate bulge from mass on small bowel capsule endoscopy. Gastrointest Endosc 2011; 74: 1067-1074

[19] Saurin JC, Delvaux M, Vahedi K et al. Clinical impact of capsule endoscopy compared to push enteroscopy: 1-year follow-up study. Endoscopy 2005; 37: 318-323

[20] Gralnek IM, Defranchis R, Seidman E et al. Development of a capsule endoscopy scoring index for small bowel mucosal inflammatory change. Aliment Pharmacol Ther 2008; 27: 146-154

[21] Gal E, Geller A, Fraser G et al. Assessment and validation of the new capsule endoscopy Crohn's disease activity index (CECDAI). Dig Dis Sci 2008; 53: 1933-1937

[22] Koffas A, Laskaratos FM, Epstein O. Non-small bowel lesion detection at small bowel capsule endoscopy: a comprehensive literature review. World J Clin Cases 2018; 6: 901-907 
[23] Li X, Chen H, Dai ] et al. Predictive role of capsule endoscopy on the insertion route of double-balloon enteroscopy. Endoscopy 2009; 41: 762-766

[24] Ponte A, Pinho R, Rodrigues A et al. Review of small-bowel cleansing scales in capsule endoscopy: A panoply of choices. World J Gastrointest Endosc 2016; 8: 600-609

[25] Yung DE, Boal Carvalho P, Giannakou A et al. Clinical validity of flexible spectral imaging color enhancement (FICE) in small-bowel capsule endoscopy: a systematic review and meta-analysis. Endoscopy 2017; 49: 258-269
[26] Read A], Rice MD, Conjeevaram HS et al. A deeper look at the small bowel: training pathways in video capsule endoscopy and device-assisted enteroscopy. Dig Dis Sci 2018; 63: 2210-2219

[27] Koffas A, Laskaratos FM, Epstein O. Training in video capsule endoscopy: current status and unmet needs. World J Gastrointest Endosc 2019; 11: 395-402

[28] lakovidis DK, Koulaouzidis A. Automatic lesion detection in capsule endoscopy based on color saliency: closer to an essential adjunct for reviewing software. Gastrointest Endosc 2014; 80: 877-883 